\title{
A CHARACTERIZATION OF THE SEMIGROUP OF MATRIX UNITS
}

\author{
BERNARD R. GELBAUM and STEPHEN SCHANUEL
}

(Received 1 July 1979)

Communicated by J. B. Miller

\begin{abstract}
Let $I$ be a set and let $\mathscr{Q}(I)$ denote the set consisting of the 0 matrix over $I \times I$ and the matrix units over $I \times I$. Then for $x, z$ in $\mathscr{U}(I)$ and $x \neq 0 \neq z, x y z \neq 0$ has precisely one solution $y$. This and several other statements are shown to be equivalent characterizations of $\mathscr{U}(I)$ regarded as a semigroup with zero.
\end{abstract}

1980 Mathematics subject classification (Amer. Math. Soc.): 20 M 10.

Keywords: Semigroups with zero, matrix units, categorical semigroups.

\section{Introduction}

This research arose in connection with an investigation of simple Banach algebras. The authors are indebted to Professors Ching Chou and Kenneth Magill for their contributions and, in some instances, essentially collaborative efforts.

The principal result centers on the well-known fact that the semigroup $\mathscr{U}(I)$ consisting of the 0 matrix and the set of all matrix units (each a matrix with precisely one nonzero entry and that entry 1$)$ for an arbitrary index set $I$ enjoys the property: if $x \neq 0 \neq z$ are in $\mathscr{U}(I)$ there is precisely one $y$ in $\mathscr{U}(I)$ such that $x y z \neq 0$. This and three other properties are shown to be equivalent characterizations of $\mathscr{U}(I)$ as a semigroup with zero.

\section{The characterization}

For an arbitrary index set $I, \mathscr{U}(I)$ denotes the semigroup $(I \times I) \cup\{0\}$ where, for $i, j, k, l$ in $I,(i, j) \cdot(k, l)=(i, l)$ if $j=k$ and $=0$ otherwise, and where 
$0 \cdot(i, j)=(i, j) \cdot 0=0$. For an arbitrary semigroup $P$ with zero $(0), P^{*}$ denotes the set $P \backslash\{0\}$. If $\mathbf{M}$ is the set of morphisms of a small category, $\mathbf{M} \cup\{0\}$ may be regarded as a semigroup with zero in which the product of two morphisms $x$ and $y$ is defined to be their composition if they may be composed and 0 otherwise and where $0 \cdot x=x \cdot 0=0$ for all morphisms $x$. Such a semigroup with zero is called a categorical semigroup.

If $\boldsymbol{P}$ is a semigroup, $l^{1}\left(P^{*}\right)$ denotes the vector space of all functions $f: P^{*} \rightarrow \mathrm{C}$ such that $\Sigma_{p}|f(p)| \equiv\|f\|<\infty$. For $f, g$ in $l^{1}\left(P^{*}\right)$ their product may be defined by convolution:

$$
(f * g)(z)=\sum_{x y=\infty} f(x) g(y),
$$

where the sum on the right is taken to be 0 if the set $\{(x, y): x y=z\}$ is empty. With respect to this product and the norm $\|\ldots\|$ above, $l^{1}\left(P^{*}\right)$ is a Banach algebra. Note that if $P=\mathscr{U}(I)$, where the cardinality of $I$ is some integer $n$, then $l^{1}\left(P^{*}\right)$ is precisely the algebra of $n \times n$ matrices over $\mathbf{C}$. When $I$ is infinite, a similar interpretation is available.

THEOREM 1. Let $P$ be a semigroup with zero. Then the following are equivalent:

(a) $P$ is isomorphic to $\mathscr{U}(I)$ for some (index) set $I$;

(b) for $x, z$ in $P^{*}$ there is precisely one $y$ in $P^{*}$ such that $x y z \neq 0$;

(b') for $x$ in $P^{*}$ there is precisely one $y$ in $P^{*}$ such that $x y x \neq 0$ and for $p, r$ in $P^{*}$, where $p^{2}=p, r^{2}=r$, there is precisely one $q$ in $P^{*}$ such that $p q r \neq 0$;

(c) for $x, z$ in $P^{*}$ there is precisely one $y$ such that $x y \neq 0$ and $y z \neq 0$;

(d) $P$ is a categorical semigroup and $l^{1}\left(P^{*}\right)$ contains no proper, closed, nontrivial ideals $\left(l^{1}\left(P^{*}\right)\right.$ is simple).

Proof. The definition of $\mathscr{U}(I)$ shows that (a) implies (b). Furthermore, for $I$ given, the category of singleton subsets $\{i\}$ of $I$ as objects and maps between them as morphisms leads to a categorical semigroup $P$. The simplicity of $l^{1}\left(P^{*}\right)$ then flows from the observation that if $(i, j)$ and $(k, l)$ are in $P^{*}$ then $(k, i)(i, j)(j, l)=(k, l)$ and $(k, i)\left(i^{\prime}, j^{\prime}\right)(j, l)=0$ if $\left(i^{\prime}, j^{\prime}\right) \neq(i, j)$. Thus $(a) \Rightarrow(\mathrm{d})$. The remaining implications are proved below according to the scheme $(\mathrm{d}) \Rightarrow(\mathrm{c}) \Rightarrow(\mathrm{b}) \Rightarrow(\mathrm{a})$ and $(b) \Leftrightarrow\left(b^{\prime}\right)$ from which the theorem follows.

(b) $\Rightarrow$ (a): For $x$ in $P^{*}$ let $u=\bar{x}$ be the unique solution of $x u x \neq 0$. Then $x \bar{x} \neq 0 \neq \bar{x} x$ and the unique solution of $(\bar{x} x) w(x \bar{x}) \neq 0$ must be $\bar{x}$ whence $\bar{x} x \bar{x} x \bar{x} \neq 0$. Since $x \bar{x} x$ and $x$ are both solutions of $\bar{x} v \bar{x} \neq 0$, there obtains:

$$
\mathbf{x} \overline{\mathbf{x}} \mathbf{x}=\mathbf{x} .
$$

This equation is central to many of the following arguments. Obviously $\overline{\bar{x}}=x$.

First, $\left({ }^{*}\right)$ shows $x \bar{x}$ and $\bar{x} x$ are idempotents and thus $I$, the set of idempotents in $P^{*}$, is nonempty. The maps $\varphi: P \rightarrow \mathscr{U}(I)$ where $\varphi(0)=0, \varphi(x)=(x \bar{x}, \bar{x} x)$ if $x \neq 0$ 
and $\psi: \mathscr{U}(I) \rightarrow P$ where $\psi(0)=0, \psi((p, q))=x$ where $p x q \neq 0$ are, as follows, shown to be inverses of each other and semigroup homomorphisms: if $x \neq 0$,

$$
\psi(\varphi(x))=\psi(x \bar{x}, \bar{x} x)=x
$$

by the earlier calculations; let $\psi((p, q))=x$; then $\varphi(\psi((p, q)))=(x \bar{x}, \bar{x} x)$ and $p x q \neq 0$. Thus $y=x \bar{x}$ and $y=p=p^{2}$ both satisfy $p(y) x q \neq 0$ whence $p=x \bar{x}$, and similarly $q=\bar{x} x$. Hence $\varphi$ and $\psi$ are inverse maps.

The map $\varphi$ is a homomorphism because: if $x, y$ are in $P^{*}$ and $x y=0$ then $\bar{x} x \neq y \bar{y}$ (otherwise $0=\bar{x}(x y)=y \bar{y} y \neq 0)$ and so $\varphi(x) \cdot \varphi(y)=(x \bar{x}, \bar{x} x) \cdot(y \bar{y}, \bar{y} y)=0$; if $x y \neq 0$, then, since $x=x \bar{x} x, y=y \bar{y} y$, it follows that $0 \neq x y=x \bar{x} x y=x y \bar{y} y$ whence $\bar{x} x=y \bar{y}$ (hence also if $x y \neq 0, \overline{x y}=\bar{y} \bar{x}$ since

$$
x y(\bar{y} \bar{x}) x y=x y \bar{y} \bar{x} x y=x \bar{x} x \bar{x} x y=x y \neq 0) ;
$$

and both $u=(x y)(\overline{x y})$ and $u=x \bar{x}$ satisfy $x \bar{x} u x y=x y \neq 0$ while both $v=(\overline{x y})(x y)$ and $v=\bar{y} y$ satisfy $x y v \bar{y} y=x y \neq 0$ whence $(x y)(\overline{x y})=x \bar{x}$ and $(\overline{x y})(x y)=\bar{y} y$; consequently if $x y \neq 0, \varphi(x) \varphi(y)=(x \bar{x}, \bar{x} x)(y \bar{y}, \bar{y} y)=(x \bar{x}, \bar{y} y), \varphi(x y)=((x y)(\overline{x y})$, $(\overline{x y})(x y))=(x \bar{x}, \bar{y} y)$ and so $\varphi(x y)=\varphi(x) \varphi(y)$. In sum $\varphi: P \rightarrow \mathscr{U}(I)$ is an isomorphism and $(b) \Rightarrow(a)$.

(c) $\Rightarrow$ (b): For $x, z$ in $P^{*}$ let $\tilde{u}$ be the unique solution of the set $x u \neq 0, u x \neq 0$, let $y$ be the unique solution of the set $x v \neq 0$ and $v z \neq 0$ and let $w$ be the unique solution of the set $\tilde{u} w \neq 0$ and $w(y z) \neq 0$. Then $t=w$ and $t=x$ both satisfy: $\tilde{u} t \neq 0$ and $t y \neq 0$ whence $w=x$ and so $x y z \neq 0$. Clearly (c) implies that $x s z \neq 0$ has at most one solution and so $(c) \Rightarrow(b)$.

(d) $\Rightarrow$ (c): Let $\mathbf{C}$ be the small category such that its set of morphisms $\mathbf{M}$ and 0 constitute $P$. For $A, B$ objects in $\mathbf{C}, \mathbf{C}(A, B)$ denotes the set of morphisms associated with $A, B$ or the empty set if none such exists. Let $\mathbf{C}_{0}$ be the category the objects of which are those of $\mathbf{C}$ and where, for $A, B$ in $\mathbf{C}_{0}, \mathrm{C}_{0}(A, B)$ (三the set of morphisms associated with $A, B)$ is a singleton set if $\mathbf{C}(A, B) \neq \varnothing$, and $\mathbf{C}_{0}(A, B)=\varnothing$ otherwise. The rule of composition in $\mathbf{C}_{0}$ is: $\mathbf{C}_{0}(A, B) \cdot \mathbf{C}_{0}(C, D)=\mathbf{C}_{0}(A, D)$ if $B=C$ and both factors are not empty. Otherwise the product is 0 . If $\mathbf{M}_{\mathbf{0}}$ is the set of morphisms for $\mathbf{C}_{0}$, let $P_{0}=\mathbf{M}_{0} \cup\{0\}$ be the associated categorical semigroup. The map $F:[\mathbf{C}, \mathbf{M}] \rightarrow\left[\mathbf{C}_{0}, \mathbf{M}_{0}\right]$, where for an object $A$ of $\mathbf{C}, F(A)=A$, and for a morphism $x \in \mathbf{C}(A, B), F(x)=\mathbf{C}_{0}(A, B)$, is a functor. Regarded as a map on $\mathbf{M}, F$ may be extended to a map $P \rightarrow P_{0}$ by setting $F(0)=0$ and then to a map $l^{1}\left(P^{*}\right) \rightarrow l^{1}\left(P_{0}^{*}\right)$ by the formula

$$
F(f)\left(p_{0}\right)=\sum_{F(p)=p_{0}} f(p)
$$

where $f$ is in $l^{1}\left(P^{*}\right), p$ is in $P^{*}, p_{0}$ is in $P_{0}^{*}$. In these circumstances $F$ is a homomorphism of $P$ onto $P_{0}$ and of $l^{1}\left(P^{*}\right)$ onto $l^{1}\left(P_{0}^{*}\right)$. Furthermore since $\|F(f)\| \leqslant\|f\|$, $F$ is continuous and $\operatorname{ker}(F)$ is closed. Since $l^{1}\left(P^{*}\right)$ is simple and since $F$ is nontrivial, it follows that $\operatorname{ker}(F)$ is $\{0\}$ and that $F$ regarded as a homomorphism of $P$ onto $P_{0}$ 
is injective. However for $\mathrm{C}_{0}(A, B)$ and $\mathrm{C}_{0}(C, D)$ in $P_{0}^{*}$ there is in $P_{0}$ at most one $\mathrm{C}_{0}(X, Y)$ such that $\mathrm{C}_{0}(A, B) \mathrm{C}_{0}(X, Y) \neq 0 \neq \mathrm{C}_{0}(X, Y) \mathrm{C}_{0}(C, D)$, namely $\mathrm{C}_{0}(X, Y)=$ $\mathrm{C}_{0}(B, C)$ and so for $x, z$ in $P^{*}$ there is at most one $y$ in $P^{*}$ such that $x y \neq 0 \neq y z$.

To prove there is at least one $y$ satisfying $x y \neq 0 \neq y z$ let $\varnothing \neq J \subsetneq P^{*}$ be called an ideal if $P^{*} J \cup J P^{*} \cup P^{*} J P^{*} \subset J \cup\{0\}$. Then clearly, for $J$ an ideal in $P^{*}, l^{1}(J)$ is a nontrivial proper closed ideal in $l^{1}\left(P^{*}\right)$. Furthermore, $R \equiv\left\{x: P^{*} x=\{0\}, x \in P^{*}\right\}$ is empty or an ideal or all $P^{*}$. If $l^{1}\left(P^{*}\right)$ is simple, then $R$ is empty and for all $x$, $P^{*} x \neq\{0\}$. By the same argument, $L \equiv\left\{x: x P^{*}=\{0\}, x \in P^{*}\right\}$ is empty if $l^{1}\left(P^{*}\right)$ is simple. Thus $P^{*} x \neq\{0\} \neq x P^{*}$ for all $x$ if $l^{1}\left(P^{*}\right)$ is simple and so $P^{*} x P^{*} \neq\{0\}$ if $l^{1}\left(P^{*}\right)$ is simple. But $P^{*} x P^{*} \backslash\{0\}$ is then nonempty and, to avoid being an ideal, must be all $P^{*}$ if $l^{1}\left(P^{*}\right)$ is simple. In other words, if $u, v$ are in $P^{*}$, then $u=p v q$, for some $p, q$ in $P^{*}$.

Now let $x=p x q, p, q$ in $P^{*}$ and let $q=y z s$. Then $x=p x y z s \neq 0$ and so $x y \neq 0 \neq y z$ as required, that is, $(d) \Rightarrow(c)$.

Clearly $(b) \Rightarrow\left(b^{\prime}\right)$. Conversely, $\left(b^{\prime}\right)$ implies that the unique $y$ such that $x y x \neq 0$ gives rise to the idempotent $x y$ (see the proof of $(b) \Rightarrow(a)$ ). Thus the set $I$ of idempotents is nonempty. If $x, z$ are in $P^{*}, p=\bar{x} x$ and $r=\bar{z} z$ are in $I$ and the unique $q$ such that $p q r \neq 0$ also satisfies $x q z \neq 0$. If $x q^{\prime} z=w \neq 0$ then

$$
0 \neq w \bar{w} w=w=x q^{\prime} z\left(\overline{x q^{\prime} z}\right) x q^{\prime} z=x q^{\prime} z \bar{z} \bar{q}^{\prime} \bar{x} x q^{\prime} z
$$

and so $z \bar{z} \bar{q}^{\prime} \bar{x} x \neq 0$. But then $\bar{x} x q^{\prime} z \bar{z}=p q^{\prime} r \neq 0$ and so $q^{\prime}=q$. Thus $\left(b^{\prime}\right) \Rightarrow(b)$ and the proof of the theorem is complete.

Note. In the proof of $(d) \Rightarrow(c)$, an alternative approach to showing that for $x \neq 0 \neq z$ there is at least one $y$ satisfying $x y \neq 0 \neq y z$ is the following: For $A, B$ objects in $\mathrm{C}$ let $B \leqslant A$ signify $\mathrm{C}(B, A) \neq \varnothing$ and let $J_{B}=\{A: A$ an object in $\mathrm{C}, B \leqslant A\}$. Since $C$ is a category the presence of identity morphisms shows $B \in J_{B}$ and so $J_{B} \neq \varnothing$. Let $\tilde{J}_{B}$ be the set of all morphisms in the sets $\mathrm{C}(C, A)$ where $A \in J_{B}$ : $\tilde{J}_{B}=\left\{x: x \in \mathbf{C}(C, A), A \in J_{B}, C\right.$ an object in $\left.\mathbf{C}\right\}$. Then $\tilde{J}_{B}$ is an ideal in $P^{*}:$ if $u, v$ are in $P^{*}$ and if $y$ is in $\tilde{J}_{B}$, let $y \in \mathbf{C}(C, A)$; then if $u y \neq 0, u \in \mathbf{C}(A, D)$ and $D \geqslant B$ whence $u y \in \mathbf{C}(C, D)$ and $u y \in \tilde{J}_{B}$; if $y v \neq 0, v \in \mathbf{C}(E, C), y v \in \mathbf{C}(C, A)$ and $y v \in \tilde{J}_{B}$; since $y v \in \tilde{J}_{B}$, uyv $\in \tilde{J}_{B} \cup\{0\}$. Since $l^{1}\left(P^{*}\right)$ is simple and since $\tilde{J}_{B} \neq \varnothing$ it follows that $\tilde{J}_{B}=P^{*}$. Thus $J_{B}$ is the set of all objects in $\mathrm{C}$ and so for any $A, B, B \leqslant A$. Consequently, if $x \in \mathbf{C}(A, B), z \in \mathbf{C}(C, D)$, the set $\mathbf{C}(D, A) \neq \varnothing$ and if $y \in \mathbf{C}(D, A)$ then $x y \in \mathbf{C}(D, B)$ and $y z \in \mathbf{C}(C, A)$, that is, $x y \neq 0 \neq y z$.

\section{Complements}

If $P$ is a semigroup with zero and if $l^{1}\left(P^{*}\right)$ is simple, $P$ may fail to be isomorphic to $\mathscr{U}(I)$ for all $I$, as the following example, due to Ching Chou, shows: Let $P$ 
consist of the $2 \times 2$ matrices

$$
\left(\begin{array}{ll}
0 & 0 \\
0 & 0
\end{array}\right), x \equiv\left(\begin{array}{ll}
1 & 0 \\
1 & 0
\end{array}\right), \quad y \equiv\left(\begin{array}{ll}
0 & 1 \\
0 & 1
\end{array}\right), z \equiv\left(\begin{array}{ll}
1 & 0 \\
0 & 0
\end{array}\right) \text { and } w \equiv\left(\begin{array}{ll}
0 & 1 \\
0 & 0
\end{array}\right) \text {. }
$$

Then $P$ is a semigroup with zero, $l^{1}\left(P^{*}\right)$ is the algebra of $2 \times 2$ matrices over $\mathbf{C}$. Nevertheless $x \cdot x=x \neq 0 \neq y=x w, x z=x \neq 0 \neq w=z w$ whence $x y w \neq 0$ does not have a unique solution $y$.

The Wedderburn-Jacobson theorems on simple algebras show that if $P$ is a finite semigroup with zero and if $l^{1}\left(P^{*}\right)$ is simple then there is a $\mathscr{U}(I)$ such that $l^{1}\left(P^{*}\right)=l^{1}\left(\mathscr{U}(I)^{*}\right)$, and the cardinality of $P$ must be $n^{2}+1$ for some integer $n$. In the proof of $(\mathrm{d}) \Rightarrow(\mathrm{c})$ there emerged: $l^{1}\left(P^{*}\right)$ simple $\Rightarrow x y z \neq 0$ has at least one solution $y$ if $x \neq 0 \neq z$; and $l^{1}\left(P^{*}\right)$ simple and $x \in P^{*} \Rightarrow P x P=P$.

Let a semigroup $P$ with 0 be called simple if it cannot be decomposed nontrivially into equivalence classes $\{S\}$ again constituting a semigroup with 0 , that is if $S_{1}, S_{2}$ are two equivalence classes then there is an equivalence class $S_{3}$ such that for $x_{1}$ in $S_{1}, x_{2}$ in $S_{2}, x_{1} x_{2}$ is in $S_{3}$ and where the cardinality of $\{S\}$ and of at least one $S$ is greater than one. Alternatively, if $h: P \rightarrow Q$ is a semigroup homomorphism then either $Q=\{0\}$ or $h$ is an isomorphism.

Call a subset $J$ of $P$ an ideal if $\{0\} \neq J \neq P$ and if $P J \cup J P \subset J$. If $J$ is an ideal let $x R y$ if and only if $x=y$ or $x$ and $y$ are in $J$. Then $R$ is an equivalence relation, $J$ is an equivalence class of cardinality greater than one, if $x \notin J,\{x\}$ is an equivalence class different from $J$, and if $x_{1} R y_{1}, x_{2} R y_{2}$ then $\left(x_{1} x_{2}\right) R\left(y_{1} y_{2}\right)$, whence $R$ decomposes $P$ nontrivially into equivalence classes constituting a semigroup with $O(J)$.

On the other hand, let $G$ be a group and let $P=G \cup\{0\}$ be a semigroup with 0 in the obvious way. The equivalence relation $x R y$ if and only if $x=y=0$ or $x, y \in G$ decomposes $P$ nontrivially and so $P$ is not simple. However, $P$ contains no ideal because $G$ is a group.

If $l^{1}\left(P^{*}\right)$ is simple so is $P$. For if $\{S\}$ is a nontrivial decomposition as described above, let $\{S\}$ constitute the semigroup $Q$ (with 0 ) and let $h: P \rightarrow Q$ be the related homomorphism. Then $h$ may be extended to $H: l^{1}\left(P^{*}\right) \rightarrow l^{1}\left(Q^{*}\right)$ according to the formula $H(f)(q)=\Sigma_{h(p)=q} f(p)$. Then $\Sigma_{q}|H(f)(q)| \leqslant \Sigma_{p}|f(p)|$ and as in earlier arguments $H$ is readily shown to be a continuous homomorphism. Since $\operatorname{ker}(H) \equiv\left\{f: \Sigma_{p} f(p)=0\right\}$ is a nontrivial ideal in $l^{1}\left(P^{*}\right)$ a contradiction results. There emerges the following chain of implications:

$$
P=\mathscr{U}(I) \stackrel{[1]}{\Rightarrow} l^{1}\left(P^{*}\right) \text { is simple } \stackrel{[2]}{\Rightarrow} P \text { is simple } \stackrel{[3]}{\Rightarrow} P \text { is ideal-free }
$$


The example $P=G \cup\{0\}$ above shows that the reverse of [3] is false. There remain the related questions:

(a) Does $l^{1}\left(P^{*}\right)$ is simple imply that $l^{1}\left(P^{*}\right)$ is isomorphic to some $l^{1}\left(\mathscr{U}(I)^{*}\right)$ ? The Chou example does not resolve this question.

(b) Is the reverse of [2] true?

Department of Mathematics

SUNY/Buffalo

U.S.A. 